\title{
Modeling Biological Rhythms in Cell Populations
}

\author{
R. El Cheikh ${ }^{1,2}$, T. Lepoutre ${ }^{1,2}$, S. Bernard ${ }^{1,2 *}$ \\ ${ }^{1}$ Université de Lyon, CNRS UMR 5208, Université Lyon 1, Institut Camille Jordan \\ 43 blvd. du 11 novembre 1918, F-69622 Villeurbanne cedex, France \\ ${ }^{2}$ INRIA project-team DRACULA, INRIA-antenne Lyon-La Doua, Batiment CEI-1 \\ 66 Boulevard Niels Bohr, 69603 Villeurbanne cedex France
}

\begin{abstract}
Biological rhythms occur at different levels in the organism. In single cells, the cell division cycle shows rhythmicity in the way its molecular regulators, the cyclin dependant kinases (CDKs), modulate their activity periodically to ensure a healthy progression. In tissues, cell proliferation is driven by the circadian clock, which modulates the progression through the cell cycle along the day. The circadian clock shows endogenous rhythmicity through a robust network of transcription-translation feedback loops that create sustained oscillations. Rhythmicity is preserved in cell populations by the coordination of the clocks among cells, through rhythmic synchronization signals. Here we discuss mechanisms for generating rhythmic activities in cell populations by reviewing some of the mathematical models that deal with them. We discuss the implication of biological rhythms for tissue growth and the possible application to chronomodulated cancer treatments.
\end{abstract}

Keywords and phrases: biological rhythms, circadian clock, cell cycle, chronotherapy, delay differential equation, age-structured equations

Mathematics Subject Classification: 92B, 92C, 35L, 34E, 34N

\section{Introduction}

The notion of rhythms in cellular biology embraces many concepts. When talking about rhythms in a single cell, one can have in mind the rhythmic total concentration of proteins, or their rhythmic shuttling in and out of the nucleus. The cell division cycle and the alternation between its phases is the prime example of how rhythmicity is ubiquitous in the life of the cell. One can observe the rhythmic waves of cell cycle protein activity or mitotic activity in well synchronized proliferating cell populations. In organisms, daily rhythms are regulated by a master pacemaker, the circadian clock. The circadian clock is a single-cell autonomous oscillator with the property of being able to synchronize between cells and to be entrained by environmental cues. It orchestrates many biological processes in a rhymed way, from the cell to the organisms physiology and behavior. Here, we review some of the concepts and origins of rhythms in biology and we present some of the mathematical models describing these phenomena.

The second section is dedicated to the concept of the circadian clock and how the rhythmic activity in its core is held by an autoregulatory system of feedback loops. We introduce delayed ordinary differential equations that can serve to model feedback loops and we show how we can pass from these DDEs to

${ }^{*}$ Corresponding author. E-mail: Email: bernard@math.univ-lyon1.fr 
generic oscillatory models. Then, we give more details about the network of genes and proteins that orchestrate these feedback loops and review a mathematical model [2] that gives a simplified but clear vision about how consistent models $[2,20,32,38]$ are constructed to deal with the machinery of the circadian clock.

The third section is dedicated to the cell cycle and its molecular models. We expose briefly how mathematicians dealt with the notion of the cell cycle and its phases. We explain the concepts of biological switches and checkpoint controls and how they were used to model the transition between the phases of the cycle. Then, we give a model [39] that illustrates the latter concepts and describes the mechanism of the molecular regulators of the cell cycle $[14,21,24,40,43,47,48]$.

In the fourth section we introduce renewal equations and structured population models used to model the cell cycle. We focus our study on the growth rate and its asymptotic behavior with and without periodic control. We take a glance on the main theoretical and numerical results obtained from this kind of models and their possible applications to cancer chronotherapy.

\section{Circadian clock}

Throughout life, our body performs a lot of daily rhythmic activities like the wake-sleep phases succession, hormone production, blood pressure and body temperature. These rhythms are regulated by a master circadian clock located in the suprachiasmatic nuclei (SCN) of the hypothalamus [57]. Many studies have shed the light on the endogenous property of this clock, in the sense that its regulation activity persists also in the absence of external factors. Nevertheless, we will see later that some of these factors like light can influence the machinery of the circadian clock.

Circadian rhythms are generated at the cellular level by a finely regulated network of genes that gives the cell the ability to generate 24 hours-period oscillations. This network involves several genes and proteins and relies on multiple interactions, transcriptional as post transcriptional mechanisms. Oscillations arise from an autoregulatory negative feedback loop system in which a clock protein once activated, mostly through phosphorylation, inhibits the expression of its own gene by inactivating the transcription factor (a protein that binds to specific DNA sequences and control the genetic information sent from DNA to mRNA)[25].

\subsection{Generic models for the circadian clock}

Because of its dynamical properties, the circadian clock has been a topic of modeling for several years. Five decades ago, Wever had developed a mathematical model for the circadian clock that was able to reproduce several of its features in living organisms [54,55]. In this model, circadian oscillations are selfsustained, but can be entrained by external cues like the sun light. The sustained oscillations generated by the circadian clock can be reproduced by a nonlinear limit cycle oscillator. The van der Pol oscillator [50] is a particularly simple differential equation that can produce a stable limit cycle. The van der Pol equation describes an oscillator $y(t)$ with a nonlinear damping coefficient $\epsilon\left(y^{2}-1\right)$.

$$
\frac{\mathrm{d}^{2} y}{\mathrm{~d} t^{2}}+\epsilon\left(y^{2}-1\right) \frac{\mathrm{d} y}{\mathrm{~d} t}+y=0 .
$$

The damping term dictates the dynamics of the oscillator. The equation reduces to the harmonic oscillator when $\epsilon=0$. For positive values of $\epsilon$, the system is non conservative, and a limit cycle exists. The existence of the limit cycle is verified by noticing that when $|y|>1$, the damping is positive (the oscillator dissipates energy) and the amplitude of $y$ decreases. When $|y|<1$, the damping is negative (the system receives energy), and the amplitude of $y$ increases. The limit cycle is the trajectory for which the average energy balance is null.

This model has been influential in the circadian modeling literature $[1,13,18,19,31,44]$. However, analysis of single-cell imaging studies from the past decade suggested that cell oscillators could be sloppy, or even damped $[5,36,51-53,56]$. This is incompatible with the van de Pol model, which always produces 
(A)

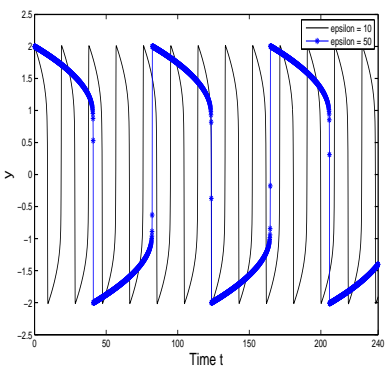

(B)

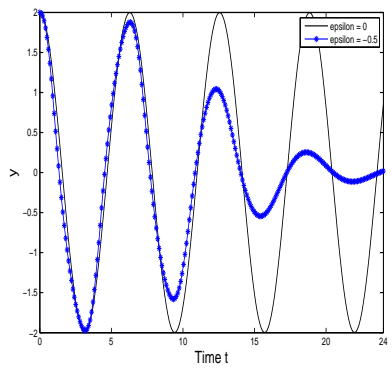

(C)

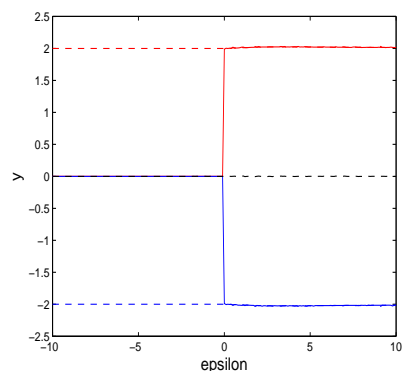

FIGURE 1. Simulations for the van der Pol system with different values of the damping coefficient $\epsilon$ : (A) Positive values for $\epsilon$ lead to a limit cycle. (B) Negative values for $\epsilon$ lead do damped oscillations. (C) Bifurcation diagram for the Van der Pol oscillator: Solid black line: stable steady state, dashed black line: unstable steady state, blue and red lines: limit cycle.

limit cycle oscillations (for positive value of $\epsilon$ ). To reproduce sloppy oscillations, a biochemical oscillator that can switch between damped and limit cycle oscillations is desirable.

One of the first and probably most popular biochemically-based models was the Goodwin model $[26,27]$. The Goodwin model refers to a class of generic molecular oscillators based on a negative feedback loop (the final product of a three-step chain of reactions inhibits the production of the first component). Later on, Ruoff et al. [45] proposed a circadian clock model inspired from the work of Goodwin. Their model dictates that a clock gene mRNA is translated into a protein that activates a transcriptional factor, which itself inhibits its own gene [25]. The original Goodwin equations [27] are

$$
\begin{aligned}
& \frac{\mathrm{d} x}{\mathrm{~d} t}=\frac{k_{0} k_{1}^{n}}{k_{1}^{n}+z^{n}}-k_{2} x, \\
& \frac{\mathrm{d} y}{\mathrm{~d} t}=k_{3} x-k_{4} y, \\
& \frac{\mathrm{d} z}{\mathrm{~d} t}=k_{5} y-k_{6} z .
\end{aligned}
$$

All equations are linear except the first one. The nonlinear term in the first equation is a negative feedback term, called a Hill function, and $z$ acts negatively on the production of $x$. The repressor $z$ can be viewed as a delayed version of the variable $x$. Standard linear stability analysis shows that three variables in the Goodwin model are necessary for a limit cycle to exist. The intermediate variable $y$ is used here to increase the delay. With a relatively high Hill coefficient $(n)$, the system can oscillate. Thus, in addition to the negative feedback loop, a delay is a necessary ingredient to obtain oscillations. High Hill coefficients are usually not biologically realistic, but if more intermediate variables or more nonlinearities are added, the Goodwin model can oscillate for smaller Hill coefficients. Either modifications to the model make it more complex to analyze, and details about intermediate steps are often unknown. Modeling circadian rhythms should take into account all the transcriptional and translational activities that are behind the oscillatory phenomena of the circadian clock, this will lead to a complex set of equations with a lot of parameters.

Instead of detailing all intermediate processes, it is tempting to introduce a "time delay" on $x$ that takes into account the time required to produce the repressor $z$. This time delay can be introduced in a clean way into the Goodwin model. Let $x$ be the amount of an activator (for example the concentration of mRNA or a protein), which produces through a linear chain process a quantity $z$, which in turn regulates $x$. We suppose that the regulator $z$ is the product of a linear chain of differential equations of length $p$, 
with kinetic parameter $a$ :

$$
\begin{aligned}
\frac{\mathrm{d} y_{1}(t)}{\mathrm{d} t} & =a\left(x(t)-y_{1}(t)\right), \\
\frac{\mathrm{d} y_{j}(t)}{\mathrm{d} t} & =a\left(y_{j-1}(t)-y_{j}(t)\right), \quad j=2, \ldots, p-1, \\
\frac{\mathrm{d} z(t)}{\mathrm{d} t} & =a\left(y_{p-1}(t)-z(t)\right) .
\end{aligned}
$$

To simplify the following, kinetic parameters of the Goodwin model were chosen to be equal $k_{i}=a$, $i=2, \ldots, 6$, but they could be different for each equation. Then, we can check that the repressor $z$ satisfies

$$
z(t)=\int_{-\infty}^{t} x(t) g_{a}^{p}(t-s) d s
$$

where the kernel $g_{a}^{p}$ is the gamma probability density function

$$
g_{a}^{p}(s)=\frac{a^{p} s^{p-1} e^{-a s}}{(p-1) !} .
$$

To see that equation (2.8) holds, we use the fact that

$$
\frac{\mathrm{d} g_{a}^{j}(t)}{\mathrm{d} t}=a\left(g_{a}^{j-1}(t)-g_{a}^{j}(t)\right), \quad j=1, \ldots, p,
$$

assuming that $g_{a}^{0}(s)=\delta_{0}(s)$ is the Dirac mass at 0 , and proceed by induction on $j$. Converting a linear activation chain into a convolution equation with a gamma kernel is called the "linear chain trick". If we re-express equation $(2.2)$ as an equation with the integral term $z(t)$, we obtain

$$
\frac{\mathrm{d} x(t)}{\mathrm{d} t}=\frac{k_{0} k_{1}^{n}}{k_{1}^{n}+\left[\int_{0}^{\infty} x(t-s) g_{a}^{p}(s) d s\right]^{n}}-k_{2} x(t) .
$$

This is a distributed delay differential equation, and this is a formulation equivalent to the set of ODEs defined by equations $(2.2,2.5-2.7)$. The gamma density can be viewed as the distribution of time required for the signal activated by $x$ to affect the production of $x$. This distribution is characterized by a mean delay $\tau=p / a$ and a variance $p / a^{2}$. The number of steps $p$ and the kinetic rates thus determine the position and the shape of the delayed distribution. When the number of steps in the linear chain $p$ and the kinetic rates $a$ go to infinity while the mean is constant, the gamma density converges to a Dirac mass at $\tau$, and $z(t)=x(t-\tau)$. Hence, feedback loops with large number of intermediate steps can be described with a discrete delay differential equation of the form

$$
\frac{\mathrm{d} x(t)}{\mathrm{d} t}=\frac{k_{0} k_{1}^{n}}{k_{1}^{n}+[x(t-\tau)]^{n}}-\alpha x(t) .
$$

This formulation of a negative feedback loop is a convenient way to capture the key role of the linear activation chain: producing a delay. This delay is necessary for a limit cycle to exist. Setting $\tau=0$ in equation (2.10) leads to a scalar ODE, which only admits monotone solutions. By using the linear chain trick for different linear chains, it is sometime possible to reduce very large systems of ODEs into single distributed delay equation with few parameters.

\subsection{Molecular models for the circadian clock}

Later on and with the help of the various discoveries in the molecular field, mathematicians started to elaborate more realistic models for the circadian clock $[2,20,25,32,38]$. The first molecular model was 


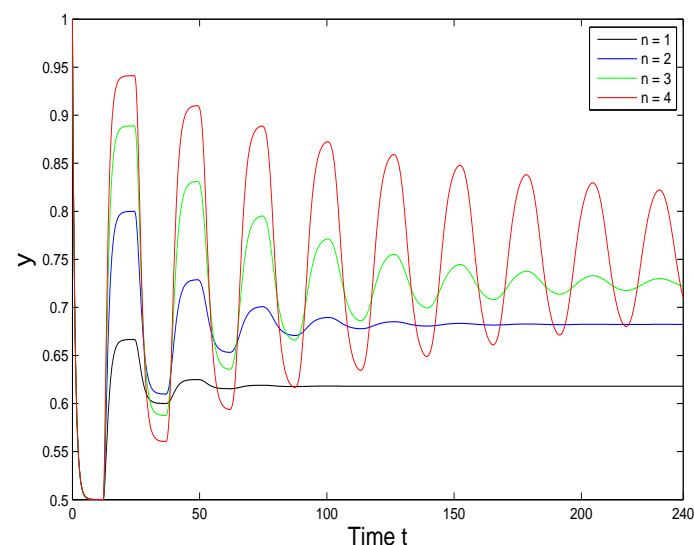

(A)

\footnotetext{
FIGURE 2. Simulations for the delayed Goodwin oscillator using equation (2.10): (A) Increasing the coefficient $n$ increases the oscillatory aspect of the model, black line: $\mathrm{n}=1$, blue line: $\mathrm{n}=2$, green line: $\mathrm{n}=3$, red line: $\mathrm{n}=4$. (B) Bifurcation diagram for the Goodwin model: Solid red line: stable steady state, dashed red line: unstable steady state, blue and green lines: limit cycle.
}

based on the fact that the protein PER inhibits its own gene and creates a negative feedback loop. A comprehensive model for the mammalian circadian clock was given by Leloup and Goldbeter [32]. They have incorporated the regulatory effects exerted on genes expression by the PER, CRY, BMAL1, CLOCK and Rev-ERB $\alpha$ proteins. In a simplified way, the model stated that the oscillations in the core of the circadian system can be generated through two negative feedback loops. The first one exerted on the expression of Per and Cry genes through the Binding of PER-CRY to the CLOCK-BMAL1 activated complex. The second one exerted by CLOCK-BMAL1 through REV-ERB $\alpha$ on the expression of Bmal1 gene. A more detailed model was given by Forger and coworkers [20], who made the distinction between the two categories of PER proteins: PER1 and PER2 and the CRY proteins: CRY1 and CRY2. They have used a slower rate of phosphorylation for PER1 because it requires more phosphorylation to bind with CRY1 or CRY2. They also used a higher coefficient of degradation for CRY2 because it is ubiquitinated more quickly than CRY1. Both models have suggested that light can enhance the transcription activity by inducing the production of PER mRNA.

In the end of this section, we review a model for the mammalian circadian clock developed by BeckerWeimann et al. [2]. This simplified (see figure 3 for a schematic explanation) model is a good example of lately developed models which take in consideration real molecular information and analyze the effect of feedback loops on the dynamics of oscillations. The model explores the negative feedback loop created by the transcription factor BMAL1/CLOCK which activates the period and chryptochrome genes(Per 1 , Per2, Cry1 and Cry2). After several hours, PER and CRY proteins downregulate their own synthesis by inhibiting BAML1/CLOCK. Once the latter protein complex is inhibited, the transcription of PER and CRY stops. Hence, BMAL1/CLOCK is no longer inhibited and the cycle restarts its process. The model also includes a positive feedback loop where Bmal1 transcription is positively regulated by PERs and CRYs.

The model is given by the following set of equations:

$$
\frac{\mathrm{d} y_{1}}{\mathrm{~d} t}=f\left(\operatorname{trans}_{P e r 2 / C r y}\right)-k_{1 d} y_{1}
$$

where:

$$
f\left(\text { trans }_{P e r 2 / C r y}\right)=\frac{\nu_{1 b}\left(y_{7}+c\right)}{k_{1 b}\left(1+\left(\frac{y_{3}}{k_{1 i}}\right)^{p}\right)+y_{7}+c} .
$$




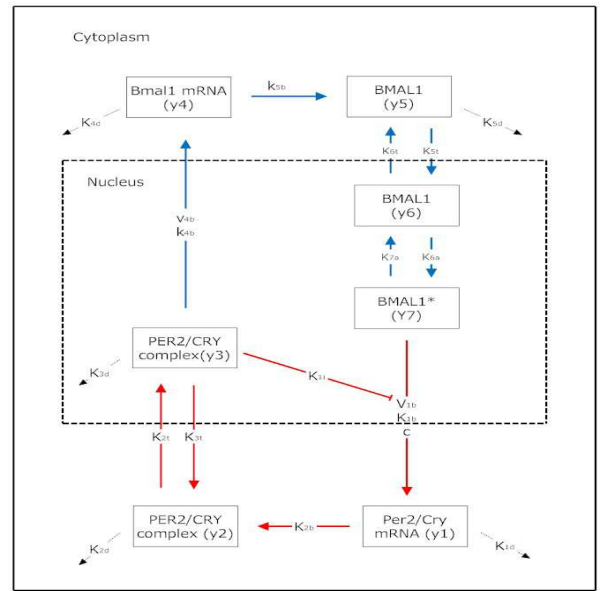

FIGURE 3. Circadian clock scheme for the model proposed by Becker-Weinmann et al. : The heterodimer BMAL1/CLOCK (y7) activates the Per and Cry genes which produce PER proteins. PER binds to CRY proteins to form a complex (y2) that is transported into the nucleus and that inhibits the activity of BMAL1/CLOCK in the nucleus, thus destructing their own source of transcription and creating the negative feedback loop. The nuclear complex PER2/CRY (y3) also activates Bmal1 transcription which implies an increase in Bmal1 mRNA (y4) and protein (y5). the nuclear BMAL1 (y6) in its activated form BMAL1* (y7) restarts the activation process of Per/Cry.

The variable $y_{1}$ represents the concentration of Per 2 or Cry mRNA which are considered to be identical, because they form a complex that is necessary for nuclear accumulation and because both are regulated by BMAL1/CLOCK and both help to stop its translational activity. As one can see from the expression of $f\left(\operatorname{trans}_{\operatorname{Per} 2 / C r y}\right)$, the variable $y_{7}$, which is an activated form of BMAL1, activates the transcription of Per2/Cry. This increases the concentration of Per2/cry mRNA $\left(y_{1}\right)$. While an increase in the variable $y_{3}$ (nuclear concentration of PER2/CRY protein), helps decreasing the rate of Per2/Cry mRNA. Notice that the coefficient $c$ plays the role of a switch-like behavior of this transcriptional regulation, $\nu_{1 b}$ is the maximal rate of Per2/Cry transcription, $k_{1 b}$ is the Michaelis constant of Per2/Cry transcription and $K_{1 d}$ is the degradation rate of Per2/Cry mRNA.

$$
\frac{\mathrm{d} y_{2}}{\mathrm{~d} t}=k_{2 b} y_{1}^{q}-k_{2 d} y_{2}-k_{2 t} y_{2}+k_{3 t} y_{3},
$$

The variable $y_{2}$ represents the concentration of the PER2/CRY complex in the cytoplasm. The coefficient $k_{2 b}$ is the rate of formation and $k_{2 d}$ is the rate of degradation of the complex. The coefficients $k_{2 t}$ and $k_{3 t}$ represent respectively the nuclear import and export of PER2/CRY, this justifies the negative sign in front of $k_{2 t}$ and the positive sign in front $k_{3 t}$.

$$
\frac{\mathrm{d} y_{3}}{\mathrm{~d} t}=k_{2 t} y_{2}-k_{3 t} y_{3}-k_{3 d} y_{3},
$$

Here, the variable $y_{3}$ represents the concentration of the PER2/CRY complex in the nucleus. This is why the signs in front of $k_{2 t}$ and $k_{3 t}$ are inverted from the previous equation. The coefficient $k_{3 d}$ represents the degradation rate of the complex.

$$
\frac{\mathrm{d} y_{4}}{\mathrm{~d} t}=f\left(\operatorname{trans}_{\text {Bmal1 }}\right)-k_{4 d} y_{4},
$$

The variable $y_{4}$ represents the concentration of Bmal1 mRNA, its rate of trancription is given by:

$$
f\left(\operatorname{trans}_{\text {Bmal1 } 1}\right)=\frac{\nu_{4 b} y_{3}^{r}}{k_{4 b}^{r}+y_{3}^{r}} .
$$




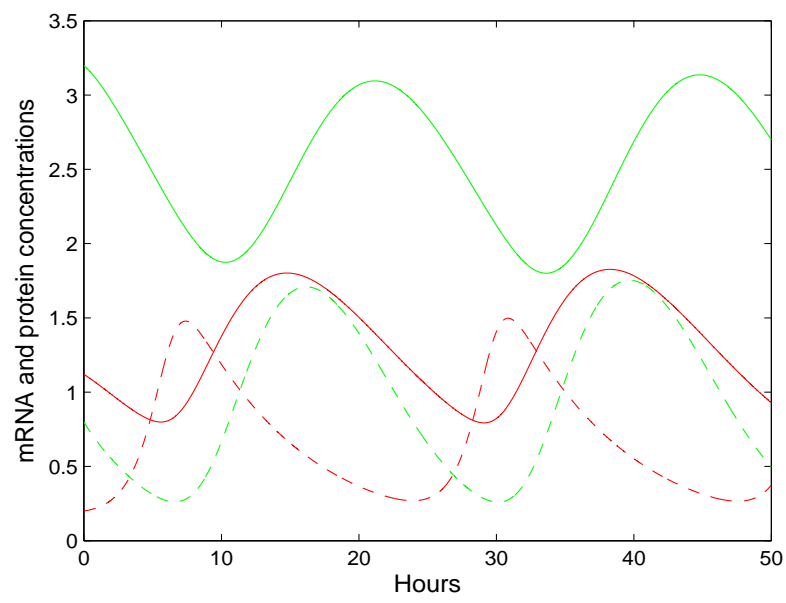

FIGURE 4. Simulation results for the Becker-Weimann et al. circadian model: Concentration of proteins and mRNAs in nM: Green line: Total BMAL1 Complex (variables: $y_{5}+y_{6}+y_{7}$ ), red solid line: PER2/CRY protein (variable $y_{3}$ ), red dashed line: Per2/Cry mRNA (variable $y_{1}$ ), green dashed line: Bmal1 mRNA (variable $y_{4}$ ). BMAL1 protein oscillates antiphasic to Per2/Cry mRNA and whith a period approximately equal to 23 hours. PER2/CRY protein oscillates with a phase delay of 7.5 hours compared to Per/cry mRNA.

One can see that the transcription rate of Bmal1 increases with rising PER2/CRY $\left(y_{3}\right)$ concentration (see figure 4, red solid line for PER2/CRY and green dashed line for Bmal1). The coefficient $k_{4 d}$ is a degradation rate. It is noteworthy here to recall that this positive action of PER2/CRY on the transcription of Bmal1 hides the repression process of Bmal1 transcription by REV-ERB $\alpha$. Hence, this latter protein is included implicitly in the model.

$$
\frac{\mathrm{d} y_{5}}{\mathrm{~d} t}=k_{5 b} y_{4}-k_{5 d} y_{5}-k_{5 t} y_{5}+k_{6 t} y_{6},
$$

The variable $y_{5}$ represents the concentration of BMAL1 cytoplasmic protein. The coefficient $k_{5 b}$ is the translation rate, $k_{5 d}$ is the degradation rate and the coefficients $k_{5 t}$ and $k_{6 t}$ represent respectively the nuclear import and export of BMAL1

$$
\frac{\mathrm{d} y_{6}}{\mathrm{~d} t}=k_{5 t} y_{5}-k_{6 t} y_{6}-k_{6 d} y_{6}+k_{7 a} y_{7}-k_{6 a} y_{6}
$$

The variable $y_{6}$ represents the concentration of BMAL1 nuclear protein. The coefficients $k_{6 d}, k_{6 a}$ and $k_{7 a}$ represent respectively, the degradation rate, the activation and deactivation of BMAL1.

$$
\frac{\mathrm{d} y_{7}}{\mathrm{~d} t}=k_{6 a} y_{6}-k_{7 a} y_{7}-k_{7 d} y_{7},
$$

The variable $y_{7}$ represents the activated form of BMAL1 (usually noted BMAL1*), which can be understood as its phosphorylated form or its combination with CLOCK.

This model shows the existence of sustained oscillations for the circadian clock for a period close to 24 hours (see figure 4, green solid line for the complex BMAL1).

\section{Cell cycle}

The cell division cycle is one of life's defining attributes by which an organism is able to reproduce and perpetuate its own species. It can be described as the process where a new born cell doubles its size, 
replicates its genetic material as well as its cellular components and gives birth to two new progeny cells who inherit all the machinery and information to repeat the process.

The cell division cycle is a very stringent process of irreversible successions, triggered by transient signals, between its four phases: G1, S(DNA synthesis), G2, and M(mitosis). The cell cycle stops its progression if these phases do not take place in the right order. In particular, DNA replication and chromosomes segregation should alternate in proliferating cells. There exists check-point controls that verify if the steps of the cycle are taking place in the right order. If a problem arises, like when the replicated chromosomes have not properly aligned on the mitotic spindle, the cell cycle will never exit mitosis. If the chromosomes are well aligned, the check point condition is satisfied, and the transition to the next phase is triggered by transient signals. These signals disappear once the cell passes to the next phase, making these transitions irreversible.

There are two main types of cell cycle models, molecular models and population models. The first one attempts to model the molecular events of the cell cycle in a single cell while the second one attempts to describe the dynamics of a population of cells, with an emphasis on cell birth and death events. The next paragraph introduces molecular models of the cell cycle.

\subsection{Molecular models of the cell cycle}

In molecular models, proteins and mRNA concentrations are often modeled with ordinary differential equations. Models are built with weakly connected modules, each module standing for one of the major checkpoints of the cell cycle. Conceptually, the cell cycle stops at each checkpoint and progression is halted until all conditions are met to raise the checkpoint. Experimental and theoretical biologists $[8,16,40,48]$ have shown that checkpoints are marked by abrupt transitions, or switches, during which specific cell cycle proteins get quickly activated or deactivated. A switch-like transition is a response to a change in concentration of a stimulus that affects the system. When the concentration of the stimulus reaches up a certain threshold, the system can switch from one state to another. There are mainly two types of switch-like transition: Continuous (sigmoidal switches) and discontinuous (bistable switches). The response in the sigmoidal switch is graded and reversible. By graded we mean that the response increases continuously with signal strength. By reversible we mean that if the signal S increases, reaches its threshold value and the system switches on to the next state, a decreasing value for $\mathrm{S}$ can bring back the system to its off state. The discontinuous responses can be separated into two kinds: The one way switch (figure $5(\mathrm{~A})$ ) and the toggle switch (figure $5(\mathrm{~B})$ ). For the one way switch, there exists a critical value $\left(S_{\text {crit }}\right)$ that the signal should attain to let the response passes to the upper state. Now, if $\mathrm{S}$ decreases the response doesn't fall back to the lower state and stays high, this is why the switch is called in this case irreversible. Notice that for $\mathrm{S}$ between 0 and $S_{\text {crit }}$, the system has two stable steady-state responses (lower and upper state) separated by an unstable one. This is why we call the switch bistable. A good example of this irreversible switch is apoptosis (a point of no return). For the toggle switch, if the response is on the upper state, and the value of $\mathrm{S}$ decreases enough, the switch will go back to the lower state. For intermediate values of $S\left(S_{\text {crit } 1}<S<S_{\text {crit } 2}\right)$, the response can be on the upper or lower state, depending on how $\mathrm{S}$ was changed. This sort of two-way discontinuous switch is often called hysteresis switch. A good example of hysteresis response is the activation of the mitosis promoting factor MPF, or the START and FINISH transitions that will be explained in the coming paragraph.

By coupling sequentially many of those bistable switches, it is possible to devise models that can follow the progression of the cell cycle $[8,24,40,48]$.

Tyson and Novak fission yeast model underlines the main molecular events behind the progress of the cell cycle. The core of this model is based on the activity of the cyclin-dependent protein kinases complexes Cdc2/Cdc13 (also called MPF or mitosis promoting factor), which are the engine needed to start DNA replication and mitosis. In this model, the cell cycle is divided into three transitions: Start, G2/M and Finish. These transitions depend on the concentration of Cdc2/Cdc13 and their enemies. If the activity of $\mathrm{Cdc} 2 / \mathrm{Cdc} 13$ is high, the cell progresses through the cell cycle; if it's low, the cell blocks its progression. Each phase transition of the cycle is regulated by specific enemies and helpers that decide 


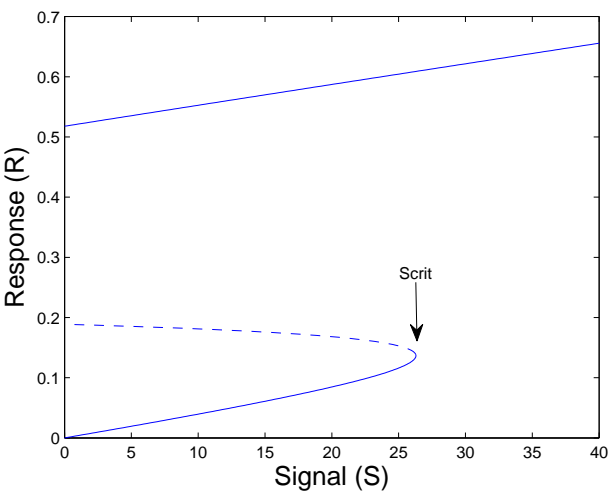

(A)

FIGURE 5. Simulations for the one way switch and toggle switch. Equations are taken from [49] (see Box 1, figure 1.e and 1.f in the reference for more details). (A) One way switch: The signal should attain the critical value "Scrit1" so that the response can pass to the upper state. If the signal decreases, the response stays on the upper state and doesn't fall back to the lower one. (B) Toggle switch: Unlike the one way switch, if the response is on the upper state and the signal decreases, the response can fall back to the lower state.

whether Cdc2/Cdc13 will win or lose. The Start transition (G1 to S) is governed by the antagonistic interaction between Cdc2/Cdc13 and their enemies Ste9 and Rum1. Ste9 targets Cdc13 to the APC core and promotes their degradation, while Rum1 binds to Cdc2/Cdc13 complexes and inhibits their activity. On the other hand, Cdc2/Cdc13 can also downregulate, by phosphorylation, the activity of Ste9 and Rum1. So what shifts the balance to $\mathrm{Cdc} 2 / \mathrm{Cdc} 13$ so that they can win and let the cell passes to the next phase? For the Start transition, there exists starter kinases that help MPF to get the upper hand and phosphorylate Ste9 and Rum1. For the Finish transition, the MPF activity should shut down to let the cell exit mitosis and enter the G1 phase. The helper molecule for this transition is the Slp1/APC complex, which promotes the degradation of $\mathrm{Cdc} 13$ and activates Ste9. Hence, the activity of the enemies will win over the activity of MPF, which shuts down and lets the cell exit mitosis. In the G2/M transition, the enemy of MPF is the tyrosine kinase WEE1, which can inactivate Cdc2. To shift the balance toward MPF, a specific phosphatase called Cdc25 removes the inhibitory effect of WEE1. Cdc25 is activated in a positive feedback by MPF.

As an example, we take the model published for the cell cycle of fission yeast [39]. The first equation describes the growth of cell mass.

$$
\frac{\mathrm{d} M}{\mathrm{~d} t}=\mu M
$$

The mass $M$ is divided by two in the EXIT phase.

The second equation describes the rate of change in $\mathrm{Cdc} 13 / \mathrm{Cdc} 2$ (named $\mathrm{Cdc} 13_{T}$ ) complex concentration

$$
\frac{\mathrm{d}\left[C d c 13_{T}\right]}{\mathrm{d} t}=k_{1} M-k_{2}^{\prime}\left[C d c 13_{T}\right]-k_{2}^{\prime \prime}[S t e 9]\left[C d c 13_{T}\right]-k_{2}^{\prime \prime \prime}[S l p 1]\left[C d c 13_{T}\right] .
$$

The first term on the right hand side assumes that the rate is proportional to the cell mass, the last three terms are the nonspecific degradation, Ste9 and Slp1-mediated degradation rates.

The third equation represents the antagonism between WEE1 and MPF through the form of the factor $k_{\text {wee }}$ (See auxiliary equations):

$$
\frac{\mathrm{d}[\operatorname{PreMPF}]}{\mathrm{d} t}=k_{w e e}\left(\left[C d c 13_{T}\right]-[\operatorname{PreMPF}]\right)-k_{25}[\operatorname{PreMPF}]-\left(k_{2}^{\prime}+k_{2}^{\prime \prime}[\text { Ste } 9]+k_{2}^{\prime \prime \prime}[\text { Slp } 1]\right)[\text { PreMPF }],
$$




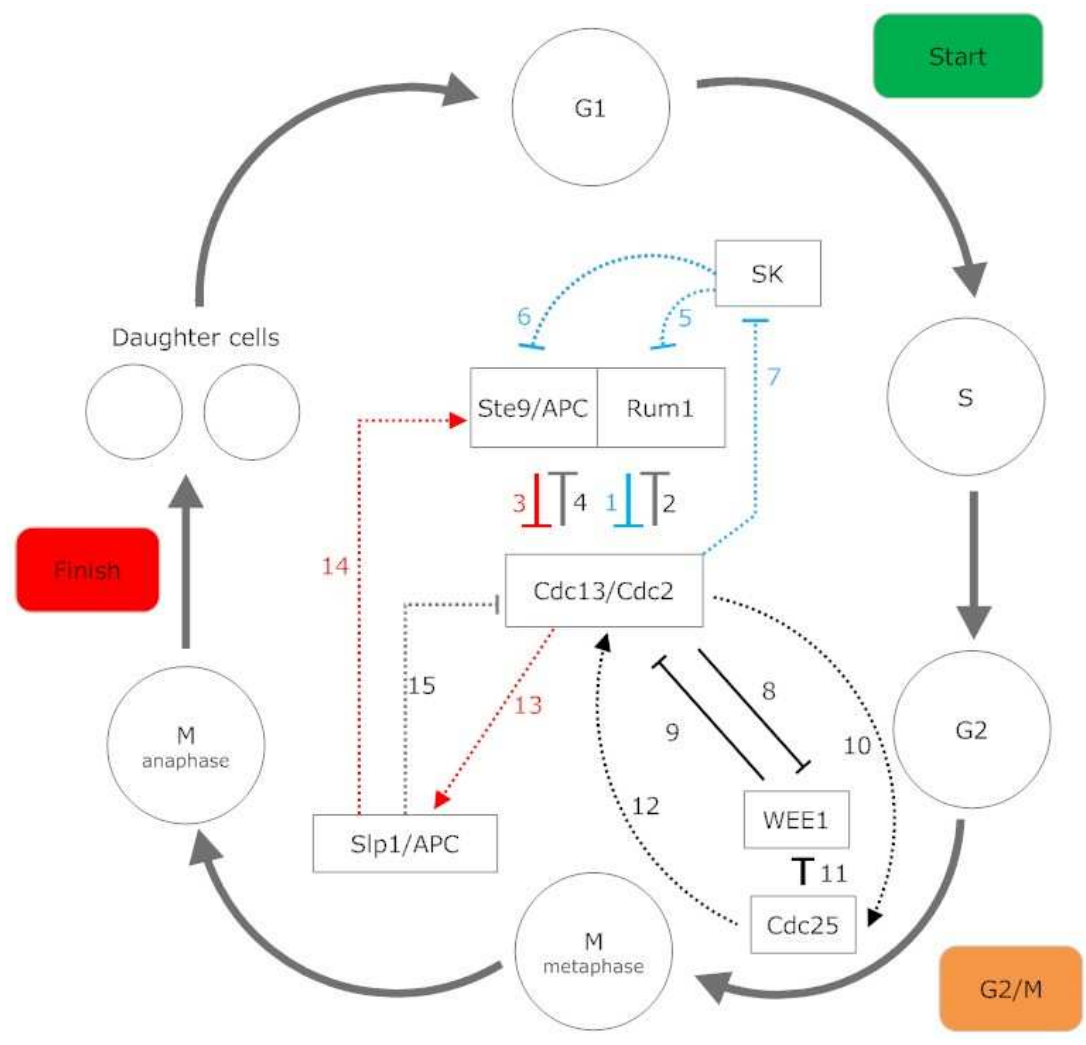

FIGURE 6. Fission yeast cell cycle: The circles represent the phases of the cell cycle, the colored rectangles show the three main transitions and the wiring diagram illustrates the antagonism between Cdc13/Cdc2 and Rum1, Ste9/APC. Green rectangle: Start transition (G1/S): Cdc2/Cdc13 and Ste9/APC, Rum1 mutual inhibition (processes: $1,2,3,4)$, Help of the starter kinase "SK" to shift the balance for Cdc2/Cdc13 by deactivating Ste9/APC, Rum1 (processes 5 and 6). Brown rectangle: G2/M transition: Mutual antagonism between Cdc2/Cdc13 and WEE1 (precesses 8 and 9), help of Cdc25 (process 12) by inactivating WEE1 (process 11), $\mathrm{Cdc} 25$ is activated in a positive feedback by $\mathrm{Cdc} 2 / \mathrm{Cdc} 13$ (process 10). Red rectangle: Finish transition $(\mathrm{M}-\dot{i}$ G1): Slp1/APC helps Ste9/APC (process 14) by inhibits the activity of Cdc2/Cdc13 (process 15). Blue and red arrows represent negative feedback loops which allow the oscillatory activity of Cdc2/Cdc14.

where PreMPF refers to the activated form of $C d c 13_{T} / C d c 2$.

The fourth equation describes the rate of change in Slp1 total concentration:

$$
\frac{\mathrm{d}[S l p 1]}{\mathrm{d} t}=k_{5}^{\prime}+\frac{k_{5}^{\prime \prime}[M P F]^{4}}{J_{5}^{4}+[M P F]^{4}}-k_{6}[S l p 1] .
$$

The first term on the right hand side is a synthesis term, the second term is a Hill type synthesis term due to $M P F$, and the last term is a degradation term.

The fifth equation describes the rate of change in the IEP enzyme activity. IEP provides the delay necessary for the chromosomes to align with the metaphase plane before they are separated at anaphase:

$$
\frac{\mathrm{d}[I E P]}{\mathrm{d} t}=\frac{k_{9}[M P F](1-[I E P])}{J_{9}+(1-[I E P])}-\frac{k_{10}[I E P]}{J_{10}+I E P} .
$$

The sixth equation describes the rate of change of Ste 9 total concentration:

$$
\frac{\mathrm{d}[S t e 9]}{\mathrm{d} t}=\frac{k_{3}^{\prime}+k_{3}^{\prime \prime}[S l p 1](1-[S t e 9])}{J_{3}+(1-[S t e 9])}-\frac{\left(k^{\prime} 4[S K]+k_{4}[M P F]\right)[S t e 9]}{J_{4}+[S t e 9]} .
$$




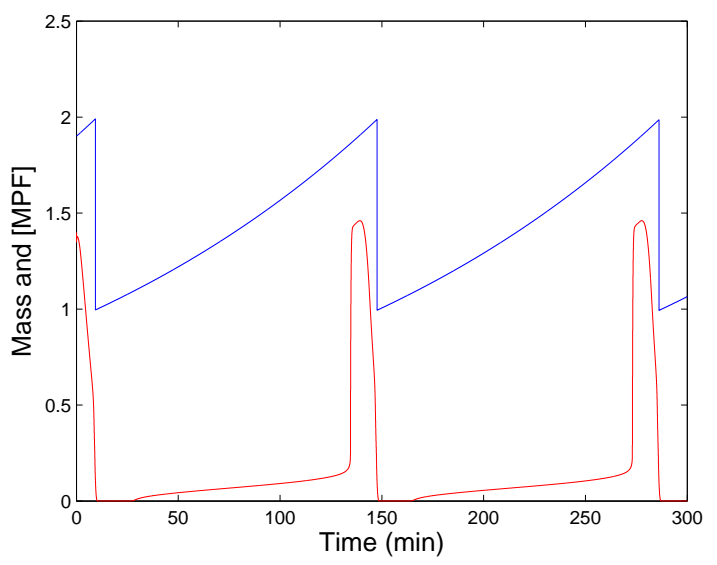

(A)

\section{FIGURE 7. Simulations for the cell cycle model porposed by Novak et al. : (A) Cell mass (Blue line) between birth and cell division is divided by two when [MPF] (Red line) decreases through 0.1 in the end of mitosis. (B) Antagonism between [Cdc13] complex (blue line) and its enemies [Ste9] (green line), [Slp1] (red line) and [Rum1] (clear blue line).}

The first term on the right hand side is an activation term and the second one represents deactivation caused by $S K$ and $M P F$.

The seventh equation describes the variation of the total concentration of Rum1:

$$
\frac{\mathrm{d}\left[R u m 1_{T}\right]}{\mathrm{d} t}=k_{11}-\left(k_{12}+k_{12}^{\prime}[S K]+k_{12}^{\prime \prime}[M P F]\right)\left[R u m 1_{T}\right] .
$$

The first term on the right hand side is a pure synthesis one, the second term represents constant degradation and degradation due to $S K$ and $M P F$ activities.

The eighth equation represents the variation of the $S K$ kinases concentration:

$$
\frac{\mathrm{d}[S K]}{\mathrm{d} t}=k_{13}[T F]-k_{14}[S K]
$$

here $T F$ is some function of the mass $M$ and on $M P F$.

The auxiliary equations are:

$$
\begin{gathered}
G(a, b, c, d)=\frac{2 a d}{b-a+b c+a d+\sqrt{(b-a+b c+a d)^{2}-4 a d(b-a)}}, \\
k_{\text {wee }}=k_{\text {wee }}^{\prime}+\left(k_{\text {wee }}^{\prime \prime}-k_{\text {wee }}^{\prime}\right) G\left(V_{\text {awee }}, V_{\text {iwee }}[M P F], J_{\text {awee }}, J_{\text {iwee }}\right), \\
k_{25}=k_{\text {wee }}^{\prime}+\left(k_{25}^{\prime \prime}-k_{25}^{\prime}\right) G\left(V_{a 25}[M P F], V_{i 25}, J_{a 25}, J_{i 25}\right), \\
\Sigma=\left[C d c 13_{T}\right]+\left[R u m 1_{T}\right]+K_{d i s s}, \\
{[\text { Trimer }]=\frac{2\left[C d c 13_{T}\right]\left[R u m 1_{T}\right]}{\Sigma+\sqrt{\Sigma^{2}-4\left[C d c 13_{T}\right]\left[\text { Rum } 1_{T}\right]}},} \\
{[M P F]=\frac{\left(\left[C d c 13_{T}\right]-[P r e M P F]\right)\left(\left[C d c 13_{T}\right]-[\text { Trimer }]\right)}{\left[C d c 13_{T}\right]},} \\
{[T F]=G\left(k_{15} M, k_{16}^{\prime}+k_{16}^{\prime \prime}[M P F], J_{15}, J_{16}\right) .}
\end{gathered}
$$




\section{Cycling cells: population models and influence on growth rates}

Several epidemiological studies have shed the light on the fact that individuals with disrupted circadian rhythms have more probability to be attained by tumorigenic diseases. For example, flight attendants and night shift workers have an increased risk of developing breast cancer. A recent study by Kubo and colleagues stated that night-shift workers are more susceptible than day workers to have prostate cancer [30]. A good explanation of this, is that the majority of tumorigenic processes arise from a disorder of the cell cycle which leads to abnormal tissue proliferation. In this section we review the results obtained on structured population models and delayed differential equations about the effect of circadian rhythms. Firstly, we will focus on linear population models and the effect of circadian rhythms will be observed as a modification of the (exponential) growth. Secondly, circadian rhythms will be represented in their simplest possible version: a periodic forcing. With such simple models, we can already observe interesting and relevant results.

\subsection{Introduction to renewal equations and structured division models}

Our typical population model is written as follows

$$
\left\{\begin{array}{l}
\partial_{t} n(t, x)+\partial_{x} n(t, x)=-d(t, x) n(t, x), \quad x>0, \\
n(t, x=0)=\int_{0}^{\infty} B(t, x) n(t, x) \mathrm{d} x .
\end{array}\right.
$$

In this model, $n(t, x)$ represents a cell density. The variable $x$ represents the age and characterizes the dynamics. Such models are called age-structured equations. Protein-structured models of the cell cycle were analyzed in $[3,15]$ without time heterogeneity. The coefficient $d(t, x)$ represents a loss term (for instance, death, but it can also contains transfer rates to other compartments) depending on age and time. The coefficient $B(t, x)$ represents the creation of new individuals at a rate $B(t, x)$. Both coefficients are periodic with respect to time, to represent the effect of circadian rhythms with a period $T$. An important example is the division model

$$
\left\{\begin{array}{l}
\partial_{t} n(t, x)+\partial_{x} n(t, x)=-[d(t, x)+K(t, x)] n(t, x), \quad x>0 \\
n(t, x=0)=2 \int_{0}^{\infty} K(t, x) n(t, x) \mathrm{d} x
\end{array}\right.
$$

In this model, the loss rate is divided into two parts: a death rate $d$ and a division rate $K$. The loss of one cell due to the term $K$ is compensated by the creation of two new cells of age $x=0$. The age $x$ is not a physiological maturity but is the chronological age since birth. Using the method of characteristics, we can easily establish the following

$$
n(t+x, x)=n(t, 0) \exp \left(-\int_{0}^{x} d(t+s, s) \mathrm{d} s\right) .
$$

The latter equation is the key to the link between structured population models and delay equations. Using the boundary condition, we can derive a Volterra-like integral equation satisfied by $n(t, 0)$

$$
n(t, 0)=\int_{0}^{\infty} B(t, x) n(t-x, 0) \exp \left(-\int_{0}^{x} d(t+s, s) \mathrm{d} s\right) .
$$

Well posedness for those models is classical in the framework of bounded coefficients and initial conditions in $L^{1}\left(\mathbb{R}_{+}\right)$(see $[42]$ for instance). 
More detailed cell cycle models can be considered. For instance, considering a cell cycle with $I$ phases (usually $I=4$ ), and following the population densities in each phase [12]. The equations reads

$$
\left\{\begin{array}{l}
\partial_{t} n_{i}(t, x)+\partial_{x} n_{i}(t, x)+\left[d_{i}(t, x)+K_{i \rightarrow i+1}(t, x) n_{i}(t, x)=0, \quad 1 \leq i \leq I \quad \text { with convention } I+1=1\right. \\
n_{i+1}(t, 0)=\int_{0}^{\infty} K_{i \rightarrow i+1}(t, x) n_{i}(t, x) \mathrm{d} x \\
n_{1}(t, 0)=2 \int_{0}^{\infty} K_{I \rightarrow 1}(t, x) n_{I}(t, x) \mathrm{d} x
\end{array}\right.
$$

As in the division models, the loss rate $d_{i}+K_{i}$ in the $i$-th equation contains two terms. A death rate $d_{i}$ and a transition rate $K_{i}$ from phase $i$ to $i+1$, which turns out to be a division rate for $i=I$. This system was studied with the aim to show that tumor growth was enhanced by circadian clock disruption. They found, using a convexity result for the dominant eigenvalue (to be introduced below) of the system that the circadian clock affects the tumor proliferation in an indirect manner. They proved that for a disrupted circadian rhythm (averaged coefficients), the dominant eigenvalue is smaller than the cases of controlled circadian rhythm (periodic coefficients). This result would imply that periodic population grows faster. This lead the authors of [12] to conclude that disrupted circadian rhythms does not enhance tumor growth directly but rather damages the healthy tissues that fight against it.

Another important class of population models in cell cycle representation are delay differential models. These models are linked to previous partial differential equations. For instance, in [10], the following discrete delay equation studied in $[4,37]$ was re-derived from a division model

$$
\frac{\mathrm{d} p(t)}{\mathrm{d} t}=-[d(t)+K(t)] p(t)+2 \sigma(t) K(t-\tau) p(t-\tau)
$$

More general delay models can be written, based on age-structured PDEs. For instance, systems where the discrete delay is replaced by a distribution of delays can be derived rigorously from division models:

$$
\frac{\mathrm{d} x(t)}{\mathrm{d} t}=-a(t) x(t)+\int_{0}^{\infty} x(t-u) b(t, u) g(u) \mathrm{d} u,
$$

The coefficients $a(t), b(t, u)$ are directly related to the coefficients of the division model with $I=2$ in the following way:

$$
\begin{aligned}
a(t) & =d_{1}(t)+K_{1}(t), \\
g(u) & =K_{2}(u) e^{-\int_{0}^{u} K_{2}(s) \mathrm{d} s}, \\
b(t, u) & =2 K_{1}(t-u) e^{-\int_{t-u}^{t} d_{2}(s, s-t+u) \mathrm{d} s} .
\end{aligned}
$$

In the following we summarize the various properties of the growth rate of such equations. Because of the close link between PDE-based and DDE-based models, results are summarized for PDE models only. We choose to write all the theorems on (4.1) but mutatis mutandis the results remain true for division models or systems.

\subsection{Asymptotic behavior with or without periodic forcing}

The quantity of interest here is the growth exponent. In the following, we will assume that coefficients satisfy conditions ensuring net growth of the population and such that the asymptotic behavior is governed by the principle eigenvalue and its associated eigenvector (examples of such conditions can be found in [12]). For simplicity, we develop the results for the renewal equation (4.1). We assume there exists a 
triple $(N, \Phi, \lambda)$, such that

$$
\left\{\begin{array}{l}
\partial_{t} N(t, x)+\partial_{x} N(t, x)=-(d(t, x)+\lambda) N(t, x) \\
N(t, x=0)=\int_{0}^{\infty} B(t, x) N(t, x) d x \\
-\partial_{t} \Phi(t, x)-\partial_{x} \Phi(t, x)+(d(t, x)+\lambda) \Phi(t, x)=B(t, x) \Phi(t, 0) . \\
\lambda \text { is a positive number, } N, \Phi \text { are postive functions } \\
\forall t, N(t+T, x)=N(t, x), \quad \Phi(t+T, x)=\Phi(t, x) \\
\frac{1}{T} \int_{0}^{T} \int_{0}^{\infty} N(t, x) \mathrm{d} x=\int_{0}^{\infty} N(t, x) \Phi(t, x) \mathrm{d} x=1 .
\end{array}\right.
$$

The last condition is just a renormalization to ensure uniqueness (note that $\int_{0}^{\infty} N(t, x) \Phi(t, x) \mathrm{d} x$ does not depend on $t$ ). Classically, such a $\lambda$ is unique and governs the growth (see [42] for detailed results on general relative entropy theory). One has, in particular,

$$
\frac{\mathrm{d}}{\mathrm{d} t} \int_{0}^{\infty} n(t, x) e^{-\lambda t} \phi(t, x) \mathrm{d} x=0, \quad \frac{\mathrm{d}}{\mathrm{d} t} \int_{0}^{\infty}\left|n(t, x) e^{-\lambda t}-\rho^{0} N(t, x)\right| \phi(t, x) \mathrm{d} x \leq 0
$$

where $\rho^{0}=\int_{0}^{\infty} n^{0}(x) \Phi(0, x) \mathrm{d} x>0$ is determined only by $n^{0}$. Conditions for existence are not the aim of the paper and the reader is asked to refer to $[12,33]$ for that purpose.

When the question of the importance of the periodic forcing arises, one of the most natural way to address it is to compare the behavior of the periodic system with a time-constant system. The first result in that direction was obtained in [12] and can be summarized as

Theorem 4.1. Assume in (4.1) that the birth rate $B$ does not depend on time. Let $\lambda_{\text {per }}$ be the growth rate of the system (4.1) and $\lambda_{s}$ be the system where $d(t, x)$ has been replaced by its arithmetical average over time

$$
d_{s}(x)=\frac{1}{T} \int_{0}^{T} d(t, x) \mathrm{d} t
$$

then the following inequality holds true

$$
\lambda_{\text {per }} \geq \lambda_{s}
$$

A little more general result was then obtained in [11] concerning the role of the birth rate $B$. Surprisingly, it leads to the apparition of a geometrical average of this rate

$$
B_{g}(x)=\exp \left(\frac{1}{T} \int_{0}^{T} \log B(t, x) \mathrm{d} t\right) .
$$

The result can be summarized as follows

Theorem 4.2. Define $\lambda_{\text {per }}$ as the growth rate of (4.1) and $\lambda_{g}$ the growth rate of (4.1) with $d$ replaced by $d_{s}$ and $B$ replaced by $B_{g}$, then the following inequality holds true

$$
\lambda_{\text {per }} \geq \lambda_{g}
$$

The introduction of different types of means can be puzzling but might be better understood by a closer look at the structure of the system. In equation (4.3), we can see that $\log B(t, x)$ and $\int_{0}^{x} d(t-x+s, s) \mathrm{d} s$ have a similar role. This mathematical result cannot be readily exploited for division or cell cycle models, at least concerning division and transition coefficients, since division rates $K$ also appear as a loss rate and a birth rate in (4.2). In this case, the averaged model cannot not be characterized as a division model because $K$ would be replaced by different functions in the PDE (by $K_{s}$ ) and in the boundary condition (by $K_{g}$ ). We end this section on averaged models by stating a negative result from [10] on comparison with arithmetical average everywhere, 


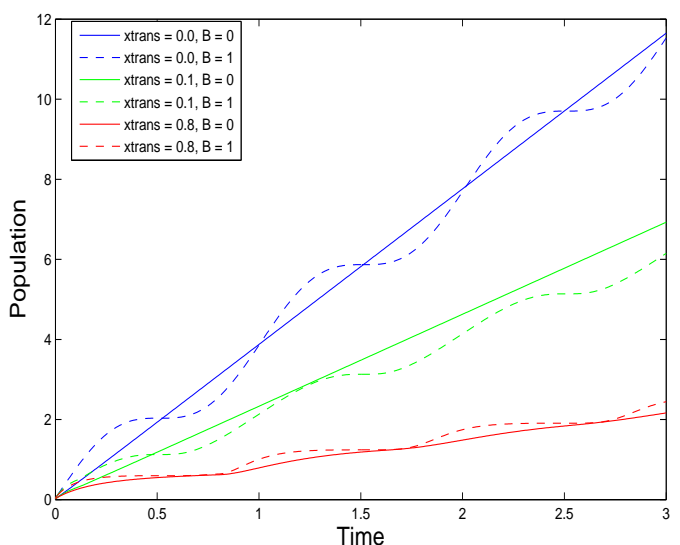

(A)

\footnotetext{
FIGURE 8. Simulations for the age structured one-phase model (logarithmic scale): (A) Influence of the transition age on the growth of the population (logarithmic scale) with and without periodic coefficients. Transition rate $K=k_{0} \mathbf{1}_{x}>x \operatorname{trans}(A+B \cos (2 \pi t / T))(\mathrm{B})$ Comparison of the growth with and without periodic coefficients. The results are coherent with theorem (3) which says then there is no general inequality between $\lambda_{\text {per }}$ and $\lambda_{s}$. In particular, one can build examples with $\lambda_{\text {per }}\left\langle\lambda_{s}\right.$ as well as $\lambda_{\text {per }}>\lambda_{s}$.
}

Theorem 4.3. Define $\lambda_{\text {per }}$ as the growth rate of (4.1) and $\lambda_{s}$ the growth rate of (4.1) replacing $d$ by $d_{s}$ and $B$ by $B_{s}$, then there is no general inequality between $\lambda_{\text {per }}$ and $\lambda_{s}$. In particular, one can build examples with $\lambda_{\text {per }}<\lambda_{s}$ as well as $\lambda_{\text {per }}>\lambda_{s}$.

This result is illustrated in figure 8. In the simulations of this figure, we tried to compare the growth of the population of cells with and without periodic forcing. The results of the simulations were coherent with the theoretical ones, in the sense that there's no general inequality between the growth with and without periodic coefficients. In figure 8(B), one can see that the curves that represent growth with and without periodic coefficient intersect and don't lead to a general comparison.

\subsection{The underlying inequality: application to chronotherapy}

The first two theorems in the previous section can be generalized to a convexity property. The following theorem was stated in [9]

Theorem 4.4. The growth rate of equation (4.1) $\lambda_{\text {per }}$ is geometrically convex with respect to $B$ and convex with respect to $d$.

This result needs explanation. Suppose we have two sets of coefficients $d^{1}, d^{2}, B^{1}, B^{2}$ with the same period $T$, and the corresponding growth rates $\lambda_{p e r}^{1}, \lambda_{p e r}^{2}$. We define an intermediate model with coefficients $d_{\theta}, B_{\theta}$, with $\theta \in(0,1)$, by

$$
\begin{aligned}
& d_{\theta}=\theta d^{1}+(1-\theta) d^{2}, \\
& B_{\theta}=\left(B^{1}\right)^{\theta}\left(B^{2}\right)^{1-\theta} .
\end{aligned}
$$

Then, from Theorem 4.4, the following inequality occurs:

$$
\lambda_{\text {per }}^{\theta} \geq \theta \lambda_{\text {per }}^{1}+(1-\theta) \lambda_{\text {per }}^{2} .
$$

Theorem 4.4 is a continuous version of the "Jensen"-based Theorem 4.2. This result can also be seen as an extension to periodic systems of an inequality given by Kingman on spectral radius of nonnegative matrices [29]. This theorem can lead to a theoretical justification of chronotherapy. Indeed, consider a drug, given every day at the same time, which side effects on healthy tissue are only represented by 
an additional death rate $d_{d r u g}\left(t-t_{a d m}, a\right)$, the parameter $t_{a d m}$ representing the effect of the time of administration of the drug. Equation (4.1) is then replaced by

$$
\left\{\begin{array}{l}
\partial_{t} n+\partial_{x} n+\left[d(t, x)+d_{d r u g}\left(t-t_{a d m}, x\right)\right] n(t, x)=0, \\
n(t, x=0)=\int_{0}^{\infty} B(t, x) n(t, x) \mathrm{d} x
\end{array}\right.
$$

and a growth rate $\lambda\left(t_{a d m}\right)$ is naturally associated. In the case of a continuous treatment, the drug induced death rate would be

$$
d_{\text {cont }}(a)=\frac{1}{T} \int_{0}^{T} d_{d r u g}(t, a) \mathrm{d} t=\frac{1}{T} \int_{0}^{T} d_{d r u g}\left(t-t_{a d m}, a\right) \mathrm{d} t_{a d m} .
$$

Again a growth rate $\lambda_{\text {cont }}$. As a consequence of the convexity of the growth rate with respect to death rates, we have

$$
\frac{1}{T} \int_{0}^{T} \lambda\left(t_{a d m}\right) \mathrm{d} t_{a d m} \geq \lambda_{\text {cont }} .
$$

An average periodic delivery is less toxic than constant delivery.

\section{Discussion}

In this review, we have discussed two important types of biological rhythms, the circadian clock and the cell cycle. The aim was to take a glance at the latest development in mathematical models attempting to describe these rhythms. Circadian clock models range from simple models used to study broad generic properties of oscillator such as period and phase, to more detailed models aiming to represent the biology of the clock realistically. Simple models are frequently represented by van der Pol or Goodwin-type oscillators $[1,13,18,19,31,44]$. More detailed models [2] try to take a deeper look on the molecular mechanism behind the circadian clock. We have seen how the core of the circadian clock is held by interlocked positive and negative feedback loops that generate robust and self sustained oscillations. Mathematical modeling is becoming nowadays a major key for constructing complex models that include a realistic representation of the biology behind the tight regulatory network of the circadian clock.

The cell division cycle has an extremely robust machinery. The precise sequential activation and inactivation of cyclins and CDKs that regulate the progression of the cycle is quite different from the smooth, periodic activation and inhibition of clock gene transcription in the circadian clock. However, the ability of the cell to repeat, potentially indefinitely, the cell cycle makes another example of a biological oscillator. Mathematical models of the cell cycle come in two flavors, single-cell molecular models, and cell population models. These two kinds of models were generally studied by mathematicians for different aims. Molecular models were often used to study the influence of some key protein complexes on the progression of the cell cycle, and look how a change in their concentration can harm a healthy cell cycle and produce damaged, smaller, bigger or dead cells after a certain number of cycles. While population models were often used to study the rate of growth of a population of cells and see the difference between a growth with and without periodic forcing.

There has recently been some interest in coupling the circadian clock with the cell cycle $[8,23,41]$. There is strong evidence that the progression through the cell cycle is gated by the circadian clock genes, resulting in daily waves of DNA synthesis and mitosis in many tissues. It has been proposed that circadian gating of the cell cycle might have evolved to protect dividing cells from harmful environment like UV, or to help coordinating metabolic activity [46]. Regardless of the implication at the molecular level, the growth rate of cell populations can vary significantly under clock control. Recent theoretical and experimental results suggest that the circadian clock plays an important role slowing down tumor growth. Moreover, the timing of daily administration can also affect the tolerance and the efficacy of anti-cancer agents. Theory suggests ways to optimize treatment schedules to adapt to the circadian clock, a strategy termed chronotherapy $[6,7,22,34,35]$, and also shows that the effect of periodic entrainment of the cell cycle leads to unexpected results. 


\section{Conclusion}

By taking into account complex interactions between the cell cycle, the circadian clock and the treatment, mathematical models are an essential tool for chronotherapy. Most of the models constructed until now lacked a realistic representation of the interaction between the circadian clock and the cell cycle. A suitable approach consists in coupling a molecular model of the cell cycle and the circadian clock within multi-phase structured equations. This way, progression through the cell cycle is conditioned by the circadian clock. G2/M transition, for instance, is gated by the protein WEE1, controlled by the clock protein BMAL1/CLOCK. Such models give an accurate view on the interdependency of the cell cycle and the circadian clock, within the cell population framework of the structured equations. Chonrontherapy arises from complex biological dynamics; we cannot avoid mathematical modeling.

\section{References}

[1] P. Achermann, H. Kunz. Modeling circadian rhythm generation in the suprachiasmatic nucleus with locally coupled self-sustained oscillators: Phase shifts and phase response curves. J Biol Rhythm, 14(6):460-468, 1999.

[2] S. Becker-Weimann, J. Wolf, H. Herzel, A. Kramer. Modeling feedback loops of the mammalian circadian oscillator. Biophys J, 87(5):3023-3034, 2004.

[3] F. Bekkal Brikci, J. Clairambault, B. Perthame. Analysis of a molecular structured population model with possible polynomial growth for the cell division cycle. Math and Comp Modelling, 47(7-8): 699-713, 2008.

[4] S. Bernard, H. Herzel. Why do cells cycle with a 24 hour period? Genome Inform Ser, 17(1):72-79, 2006.

[5] S. Bernard, D. Gonze, B. Căjavec, H. Herzel, A. Kramer. Synchronization-induced rhythmicity of circadian oscillations in the suprachiasmatic nucleus PLoS Comput Biol, 17(1):72-79, 2006.

[6] S. Bernard, B. Căjavec Bernard, F. Lévi, H. Herzel. Tumor growth rate determines the timing of optimal chronomodulated treatment schedules. LoS Comput Biol 6(3):e1000712, 2010. doi:10.1371/journal.pcbi.1000712

[7] F. Billy, J. Clairambault, O. Fercoq. Optimisation of cancer drug treatments using cell population dynamics. Math Meth and Mod in Biomed, 257-299, 2012.

[8] A. Chauhan, S. Lorenzen, H. Herzel, S. Bernard. Regulation of mammalian cell cycle progression in the regenerating liver. J Theor Biol, 283(1):103-12, 2011.

[9] J. Clairambault, S. Gaubert, T. Lepoutre. Circadian rhythm and cell population growth. Math Comput Model, 53(78):1558-1567, 2011.

[10] J. Clairambault, S. Gaubert, T. Lepoutre. Comparison of Perron and Floquet eigenvalues in age structured cell division cycle models. Math Model Nat Phenom, 4(3):183-209, 2009.

[11] J. Clairambault, S. Gaubert, B. Perthame. An inequality for the Perron and Floquet eigenvalues of monotone differential systems and age structured equations. C R Math, 345(10):549-554, 2007.

[12] J. Clairambault, P. Michel, B. Perthame. Circadian rhythm and tumour growth. C R Math, 342(1):17-22, 2006.

[13] C. Czeisler, R. Kronauer, J. Allan, J. Duffy, M. Jewett, E. Brown, J. Ronda. Bright light induction of strong (type 0) resetting of the human circadian pacemaker. science, 244(4910):1328-1333, 1989.

[14] M. Davidich, S. Bornholdt. Boolean network model predicts cell cycle sequence of fission yeast. PLoS One, 3(2):e1672, 2008.

[15] M. Doumic. Analysis of a population Model Structured by the Cells Molecular Contents. MMNP, 3(2): 121-152, 2007.

[16] J. E. Ferrell, T. Y.-c. Tsai, Q. Yang. Modeling the cell cycle: why do certain circuits oscillate? Cell, 144(6):874-85, 2011.

[17] PC. da Fonseca, J. He, EP. Morris. Molecular model of the human 26S proteasome. Mol Cell, 46(1):54-66, 2012.

[18] D. Forger, M. Jewett, R. Kronauer. A simpler model of the human circadian pacemaker. J Biol Rhythm, 14(6):533-538, 1999.

[19] D. Forger, R. Kronauer. Reconciling mathematical models of biological clocks by averaging on approximate manifolds. SIAM J Appl Math., pages 1281-1296, 2002.

[20] D. B. Forger, C. S. Peskin. A detailed predictive model of the mammalian circadian clock. Proc Natl Acad Sci USA, 100(25):14806-14811, 2003.

[21] C. Gérard, A. Goldbeter. A skeleton model for the network of cyclin-dependent kinases driving the mammalian cell cycle. Interface Focus, 1(1):24-35, 2011.

[22] S. Gery, HP Koeffler. Circadian rhythms and cancer. Cell Cycle, 9:1097-1103, 2010.

[23] C. Gérard, A. Goldbeter. Entrainment of the Mammalian Cell Cycle by the Circadian Clock: Modeling Two Coupled Cellular Rhythms. Plos Comp Biol, 8(5): e1002516.

[24] A. Goldbeter, C. Ge, C. Gérard. Temporal self-organization of the Cyclin/Cdk network driving the mammalian cell cycle. Proc Natl Acad Sci USA, 1-6, 2009.

[25] D. Gonze. Modeling circadian clocks: From equations to oscillations. Cent Eur J Biol, 6(5):699-711, 2011.

[26] B.C. Goodwin. Temporal Organization in Cells. A Dynamic Theory of Cellular Control Processes. New York: Academic Press, 1963.

[27] B.C. Goodwin. Oscillatory behavior in enzymatic control processes. Advances in Enzyme Regulation, 3:425-438, 1965. 
[28] T. Hunt. The Life Scientific, BBC Radio 4 podcast, 13/12/2011.

[29] J.F.C. Kingman. A convexity property of positive matrices. Quart. J. Math. Oxford, (2)12:283-284, 1961.

[30] T. Kubo, K. Ozasa, K. Mikami, K. Wakai, Y. Fujino, Y. Watanabe, T. Miki, M. Nakao, K. Hayashi, K. Suzuki, et al. Prospective cohort study of the risk of prostate cancer among rotating-shift workers: findings from the japan collaborative cohort study. Am J Epidemiol, 164(6):549-555, 2006.

[31] H. Kunz, P. Achermann. Simulation of circadian rhythm generation in the suprachiasmatic nucleus with locally coupled self-sustained oscillators. J Theor Biol, 224(1):63-78, 2003.

[32] J.-C. Leloup, A. Goldbeter. Toward a detailed computational model for the mammalian circadian clock. Proc Natl Acad Sci USA, 100(12):7051-7056, 2003.

[33] T. Lepoutre. Analysis and modelling of growth and motion phenomenon from biology. PHD in applied mathematics. Université Pierre et Marie Curie Paris (France), 2007-2009.

[34] F. Lévi. Circadian chronotherapy for human cancers. The Lancet Oncology, 2(5), 307-315, 2001, doi:10.1016/S14702045(00)00326-0

[35] F. Lévi. Cancer chronotherapy. J of Pharmacy and Pharmacol, 51(8), 891-898, 1999.

[36] E.S. Maywood, A.B. Reddy, G.K.Y. Wong, J.S. O’Neill, J.A. O’Brien, D.G. McMahon, A.J. Harmar, H. Okamura, M.H. Hastings. Synchronisation and maintenance of timekeeping in suprachiasmatic circadian clock cells by neuropeptidergic signaling. Curr Biol, 16:599-605, 2006.

[37] M.C. Mackey. Unified hypothesis for the origin of aplastic anemia and periodic hematopoiesis. Blood, 51(5):941-56, 1978.

[38] H. Mirsky, A. Liu, D. Welsh, S. Kay, F. Doyle. A model of the cell-autonomous mammalian circadian clock. Proc Natl Acad Sci USA, 106(27):11107-11112, 2009.

[39] B. Novak, Z. Pataki, A. Ciliberto, J.J. Tyson. Mathematical model of the cell division cycle of fission yeast. Chaos, 11(1):277-286, 2001

[40] B. Novak, J.J. Tyson. A model for restriction point control of the mammalian cell cycle. J Theor Biol, 230(4):563-579, 2004.

[41] B.F. Pando, A. van Oudenaarden. Coupling cellular oscillators-circadian and cell division cycles in cyanobacterial cells. Curr Opin Genet Dev, 20:1-6, 2010.

[42] B. Perthame. Transport equations in biology. Birkhauser, 2007.

[43] J. R. Pomerening, E. D. Sontag, J. E. Ferrell. Building a cell cycle oscillator: hysteresis and bistability in the activation of Cdc2. Nat Cell Biol, 5(4):346-51, 2003.

[44] K. Rompala, R. Rand, H. Howland. Dynamics of three coupled van der Pol oscillators with application to circadian rhythms. Commun Nonlinear Sci, 12(5):794-803, 2007.

[45] P. Ruoff, C.M. M Vindjevik, L. Rensing. The Goodwin model simulating the effect of light pulses on the circadian sporulation rhythm of Neurospora crassa. J. Theor. Biol., 209:29-42, 2001.

[46] S. Sahar, P. Sassone-Corsi. Circadian rhythms and memory formation: regulation by chromatin remodeling. Front Mol Neurosci, 5-37, 2006. Published online 2012 March 26. doi: 10.3389/fnmol.2012.00037.

[47] M. Swat, A. Kel, H. Herzel. Bifurcation analysis of the regulatory modules of the mammalian G1/S transition. Bioinformatics, 20(10):1506-1511, 2004.

[48] J.J. Tyson, B. Novak. Temporal organization of the cell cycle. Curr Biol 18, R759-R768, 2008.

[49] J.J. Tyson, K.C. Chen, B. Novak. Sniffers, buzzers, toggles and blinkers: dynamics of regulatory and signaling pathways in the cell. Curr Op in Cell Biol, 15:221-231, 2003.

[50] B. Van der Pol, J. Van der Mark. Frequency demultiplication. Nature, 120:363-364, 1927.

[51] A.B. Webb, N. Angelo, J.E. Huettner, E.D. Herzog. Intrinsic, nondeterministic circadian rhythm generation in identified mammalian neurons. Proc Natl Acad Sci USA, 106(38):16493-16498, 2009.

[52] D. Welsh, J. Takahashi, S. Kay. Suprachiasmatic nucleus: cell autonomy and network properties. Ann Rev Physiol, $72: 551-577,2010$.

[53] P.O. Westermark, D.K. Welsh, H. Okamura, H. Herzel. Quantification of Circadian Rhythms in Single Cells. PLoS Comput Biol, 5(11):e1000580, 2009.

[54] R. Wever. Zum Mechanismus der Biologischen 24-Stunden-Periodik. Biol Cybern, 1(4):139-154, 1962.

[55] R. Wever. Zum Mechanismus der Biologischen 24-Stunden-Periodik II. Biol Cybern, 1(6):213-231, 1963.

[56] S. Yamaguchi, H. Isejima, T. Matsuo, R. Okura, K. Yagita, M. Kobayashi, H Okamura, H. Synchronization of cellular clocks in the suprachiasmatic nucleus. Science, 302:1408-1412, 2003.

[57] E. E. Zhang, S. A. Kay. Clocks not winding down: unravelling circadian networks. Nat Rev Mol Cell Biol, 11(11):764776,2010 . 


\begin{tabular}{|c|c|c|c|}
\hline Parameter & Value & Parameter & Value \\
\hline$n_{1 b}$ & 9 & $n u_{4 b}$ & 3.6 \\
$k_{1 b}$ & 1 & $k_{4 b}$ & 2.16 \\
$k_{1 i}$ & 0.56 & $r$ & 3 \\
$c$ & 0.01 & $k_{4 d}$ & 0.75 \\
$p$ & 8 & $k_{5 b}$ & 0.24 \\
$k_{1 d}$ & 0.12 & $k_{5 d}$ & 0.06 \\
$k_{2 b}$ & 0.3 & $k_{5 t}$ & 0.45 \\
$q$ & 2 & $k_{6 t}$ & 0.06 \\
$k_{2 d}$ & 0.05 & $k_{6 d}$ & 0.12 \\
$k_{2 t}$ & 0.24 & $k_{6 a}$ & 0.09 \\
$k_{3 t}$ & 0.02 & $k_{7 a}$ & 0.003 \\
$k_{3 d}$ & 0.12 & $k_{7 d}$ & 0.09 \\
\hline
\end{tabular}

TABLE 1. Parameter values for the circadian clock model

\begin{tabular}{|c|c|c|c|c|c|c|c|}
\hline Parameter & Value & Parameter & Value & Parameter & Value & Parameter & Value \\
\hline$k_{1}$ & 0.03 & $J_{5}$ & 0.3 & $k_{\text {diss }}$ & 0.001 & $V_{i 25}$ & 0.25 \\
$k_{2}^{\prime}$ & 0.03 & $k_{7}$ & 1 & $k_{13}$ & 0.1 & $J_{a 25}$ & 0.01 \\
$k_{2}^{\prime \prime}$ & 1 & $k_{8}$ & 0.25 & $k_{14}$ & 0.1 & $J_{i 25}$ & 0.01 \\
$k_{2}^{\prime \prime \prime}$ & 0.1 & $J_{7}$ & 0.001 & $k_{15}$ & 1.5 & $k_{w e e}^{\prime}$ & 0.15, \\
$k_{3}^{\prime}$ & 1 & $J_{8}$ & 0.001 & $k_{16}^{\prime}$ & 1 & $k_{w e e}^{\prime \prime}$ & 1.3, \\
$k_{3}^{\prime \prime}$ & 10 & $k_{9}$ & 0.1 & $k_{16}^{\prime \prime}$ & 2 & $k_{25}^{\prime}$ & 0.05 \\
$J_{3}$ & 0.01 & $k_{10}$ & 0.04 & $J_{15}$ & 0.01 & $k_{25}^{\prime \prime}$ & 5 \\
$k_{4}^{\prime}$ & 2 & $J_{9}$ & 0.01 & $J_{16}$ & 0.01 & $\mu$ & 0.005 \\
$k_{4}$ & 35 & $J_{10}$ & 0.01 & $V_{\text {awee }}$ & 0.25 & $k_{6}$ & 0.1 \\
$J_{4}$ & 0.01 & $k_{11}$ & 0.1 & $V_{\text {iwee }}$ & 1 & $k_{12}^{\prime \prime}$ & 3 \\
$k_{5}^{\prime}$ & 0.005 & $k_{12}$ & 0.01 & $J_{\text {awee }}$ & 0.01 & $V_{a 25}$ & 1 \\
$k_{5}^{\prime \prime}$ & 0.3 & $k_{12}^{\prime}$ & 1 & $J_{\text {iwee }}$ & 0.01 & & \\
\hline
\end{tabular}

TABLE 2. Parameter values for the cell cycle model. All parameters have units $\mathrm{min}^{-1}$, except the $J i_{s}^{\prime}$ and $k_{\text {diss }}$ which are dimensionless constants. 